EPJ Web of Conferences 32, 04001 (2012)

DOI: $10.1051 /$ epjconf/20123204001

(C) Owned by the authors, published by EDP Sciences, 2012

\title{
Summary of papers on technology
}

B. Plaum ${ }^{1}$

Institut für Plasmaforschung, Universität Stuttgart D-70569 Stuttgart, Germany

e-mail: plaum@ipf .uni-stuttgart.de

Abstract. The contributions on technology are summarized

\section{Gyrotrons}

The results from the Russian ITER gyrotron development (G. Denisov) show stable $960 \mathrm{~kW}$ operation over pulse lengths of $400 \mathrm{sec}$. The reproducability and the similarity of the parameters of two test tubes where emphazized. Further development focuses on multi-frequency operation, where the vacuum window is identified as the main challenge. Several approaches for broadband windows were presented.

From CPI the status of gyrotron develpment was presented by K. Felch. The $110 \mathrm{GHz}$ 1.2 MW Gyrotron for General Atomics achieved the design power with an efficiency of $41 \%$ in short pulse operation and is shipped to GA for testing with 10 s pulses. Another development is for a $170 \mathrm{GHz} 1 \mathrm{MW}$ gyrotron for ORNL.

The status of the European $2 \mathrm{MW}$ coaxial gyrotron ITER was reported by S. Kern. After experiences with the first CW prototype, improvements were done for the electron gun, the launcher and the beam tunnel. These changes suppressed the parasitic oscillations and improved the Gaussian content of the output beam to $96 \%$. The refurbished CW prototype was tested sucessfully for 4 days until an absborber failure permanently damaged the tube and terminated the experiments. If the $2 \mathrm{MW}$ project will be terminated in favor of an $1 \mathrm{MW}$ conventional gyrotron is still to be decided.

In the ITER session, the JAEA activities for developing a dual frequency $(170 \mathrm{GHz}$ and $137 \mathrm{GHz}$ ) gyrotron where presented by Y. Oda along with test results of the ITER type transmission line. During the tests, a power of $600 \mathrm{~kW}$ was obtained for $160 \mathrm{~s}$.

The historic overview of the gyrotron development was presented by G. Nusinovich as an honorary lecture.

Further gyrotron related topics focused on a better understanding and modelling of the interactions of the electron beam and the microwave. This is important especially for the suppression of parasitic oscillations, which were observed in several MW gyrotrons and which can decrease the performance.

On the modelling side, the EURIDICE code package was presented and benchmarked against other code packages (K.A. Avramides). More specific, the suppression of spurious oscillations by using dielectric ring loaded aperiodic beam tunnels was investigated (J.G. Chelis).

A system for time- and frequency dependent characterization of gyrotron radiation was presented (A. Schlaich). It allows to observe transient phenomena (e.g. the frequency change at the beginning of the operation) as well as parasitic oscillations. It therefore provides a tool for further optimizing the interaction of the electron beam and the microwave.

Another advanced gyrotron topic is the multifrequency operation. While the Russian activities in the development of multi-frequency gyrotron mode coverters (as presented by 
G. Denisov) sound very promising, the development of multi-frequency diamond windows is still an open issue. Brewster windows require large disk sizes of CVD Diamond, which are not yet available. The principal feasibility was, however, investigated by simulating the brazing and cooling process and doing an FEM stress analysis, and suitable parameters were found (G. Aiello). The 3.6 MW power supply for the $170 \mathrm{GHz}$ ECRH system in K-Star was presented Eun-yong Shim.

\section{Diplexers}

Several papers reporting about the development of resonant diplexers were submitted. The diplexers are four-port devices with 2 input- and 2 output ports. They can be used for many applications, like fast switching between output channels (by electrically tuning the gyrotron frequency), slow switching (by mechanically tuning the resonator), power splitting, power combination and ECE diagnostics with the ECRH antenna. The overview talk (W. Kasparek) described the principle, the realisation and the results of high power experiments at the ECRH facilities of W7-X and Asdex-Upgrade. The mirror control for tuning the resonator was presented by N. Doelman. Investigations of the mode filtering capabilities of a diplexer designed and built in Japan were presented by Y. Oda. Measurements on a diplexer for FTU were reported by O. D'Arcangelo.

\section{Overall systems}

The complete 2 MW $140 \mathrm{GHz}$ ECRH system of the HL-2A tokamak was presented (M. Huang), the current status of the development of the ECRH system for ITER was presented by C. Darbos.

Furthermore, some more general remarks about large scale ECRH systems were given by V. Erckmann. Based on the experiences with the development of the ECRH system for W7-X several issues were discussed. The gyrotrons in the MW class are still designed for each customer separately, preventing a real series production. One prossible solution would be slightly relaxed gyrotron requirements (e.g. frequencies slightly different from the ideal) with the goal to have standard gyrotron specifications suitable for a large number of fusion experiments. This would reduce development costs and enable a real international competition of manufactureres. In addition, the importance of diplexers and the advantages of remote steering launchers, especially for DEMO, were outlined.

\section{Further topics}

A method for synthesizing waveguide components was presented by D.I. Sobolev. The algorithm does two propagations, one from the input (with the starting field pattern) in forward direction. The second propagation starts with the desired field pattern at the output and goes in backward direction. For an ideal device, the two field amplitudes for both propagations are identical. By comparing the amplitudes at different locations, a geometrical perturbation is introduced and the propagation is repeated. The algorithm converges very fast with good results.

The characterization of spurious modes in $\mathrm{HE}_{11}$ transmission lines with a 5-port coupler was presented by B. Plaum. By integrating leaky wave antennas into a mitre-bend, one can sample the field at 5 positions, which allows the detection of the powers of 5 modes. A formalism for obtaining the mode amplitudes from the coupler channels was developed and experimental results confirm the behavior of the device.

For the ITER ECRH system, D. Strauss gave an overview of the current status of the upper laucher and R.A. Olstad presented the development of transmission line components at General Atomics. 